\begin{tabular}{|c|c|}
\hline Title & $\begin{array}{l}\text { An Europium (III) Luminophore with Pressure Sensing Units: Effective Back Energy Transfer in Coordination } \\
\text { Polymers with Hexadentate Porous Stable Networks }\end{array}$ \\
\hline Author(s) & $\begin{array}{l}\text { Hasegawa, Y asuchika; Sawanobori, Takuya; Kitagawa, Y uichi; Shoji, Sunao; Fushimi, Koji; Nakasaka, Y uta; Masuda, } \\
\text { Takao; Hisaki, Ichiro }\end{array}$ \\
\hline Citation & $\begin{array}{l}\text { ChemPlusChem, 85(9), 1989-1993 } \\
\text { https://doi.org/10.1002/cplu.202000513 }\end{array}$ \\
\hline Issue Date & $2020-09$ \\
\hline Doc URL & http:/hdl .handle.net/2115/82575 \\
\hline Rights & $\begin{array}{l}\text { This is the accepted version of the following article: ChemPlusChem, which has been published in final form at } \\
\text { https://hemistry-europe.onlinelibrary.wiley.com/doi } / 10.1002 / \text { plu. } 202000513 \text {. }\end{array}$ \\
\hline Type & article (author version) \\
\hline File Information & hase-02sens ms5.pdf \\
\hline
\end{tabular}

Instructions for use 


\title{
An Europium(III) Luminophore with Pressure-sensing Units: Effective Back Energy Transfer in Coordination Polymers with Hexadentate Porous Stable Networks
}

\author{
Yasuchika Hasegawa, ${ }^{*}$ [a], [b] Takuya, Sawanobori, ${ }^{[b]}$ Yuichi Kitagawa, ${ }^{[a], ~[b] ~ S u n a o, ~ S h o j i, ~[b] ~ K o j i ~}$ \\ Fushimi, ${ }^{[b]}$ Yuta Nakasaka, ${ }^{[b]}$ Takao Masuda, ${ }^{[b]}$ Ichiro Hisaki[c]
}

\begin{abstract}
A luminescent Eu'll coordination polymer with $\mathrm{O}_{2}$-sensing units under air, Eu'II-hcpt (hcpt: 2,3,6,7,10,11-hexakis(4-carboxyphenyl)triphenylene), is reported. The hexadentate carboxylic acids in hcpt ligands play an important role in the formation of tight-packed three-dimensional networks in Eu'll-hcpt, producing hyper thermostable ability (decomposition temperature $=420^{\circ} \mathrm{C}$ ). The threedimensional porous network promotes bright luminescence (4f-4f emission quantum yield $=70 \%)$. The emission lifetime of Eu'II-hcpt under vacuum $(0.86 \mathrm{~ms})$ was twice as large as that under $\mathrm{O}_{2}(0.48 \mathrm{~ms}$, 1 atom:101.3 kPa). The Arrhenius analysis of the emission decay profile indicates that the back energy transfer (BEnT) from the emitting level of the Eull ion to the excited $T_{1}$ state of the hcpt ligand should be activated at room temperature. The gradual decrease of emission lifetime is caused by the BEnT process in Eu'II-hcpt. In this study, an advanced pressure-sensitive luminophore is demonstrated.
\end{abstract}

In the fields of aerodynamic science and engineering, pressuresensitive paints (PSPs) are promising molecules to accurately measure the physical parameters of a material surfaces. ${ }^{[1]}$ The luminescent PSPs provide multi-dimensional pressure data for the mapping of surfaces, whereas optoelectronic sensors analyze only single points on a surface. ${ }^{[2]}$ There are high expectations that the luminescent PSPs for surface measurements will overcome this intrinsic limitation of optoelectronic devices. Recent highspeed aircraft and automobiles have been designed using luminescent PSP techniques in wind-tunnel experiments. ${ }^{[3]}$

The pressure-sensing ability of luminescent PSPs is based on the excited quenching process of phosphorescent materials in an oxygenated environment. The electronic configuration of oxygen molecules $\left(\mathrm{O}_{2}\right)$ is normally a spin-active triplet state, which promotes effective luminescence quenching of the excited triplet state $\left(T_{1}\right)$ of phosphorescent materials. ${ }^{[4]}$ Luminescent metal complexes exhibit effective metal-to-ligand charge transfer (MLCT) phosphorescence at room temperature. Various types of phosphorescent metal complexes with $\mathrm{O}_{2}$-sensing properties have been reported to date..$^{[5]}$ Teets described a phosphorescent

[a] Prof. Dr. Y. Hasegawa, Dr. Y. Kitagawa

Institute for Chemical Reaction Design and Discovery (WPIICReDD), Hokkaido University, Sapporo, Hokkaido 001-0021, Japan

E-mail: hasegaway@eng.hokudai.ac.jp

[b] Prof. Dr. Y. Hasegawa, T. Sawanobori, Dr. Y. Kitagawa, Dr. S. Shoji, Dr. K. Fushimi,

Faculty of Engineering, Hokkaido University

Kita-13, Nishi-8, Sapporo, Hokkaido, 060-8628, Japan

[c] Prof. Dr. I. Hisaki

Graduate School of Engineering Science, Osaka University

1-3 Machikaneyama, Toyonaka, Osaka 560-8531, Japan

Supporting information for this article is given via a link at the end of the document.
IrIII complex with fluorescent BODIPY for raiometirc $\mathrm{O}_{2}$-sensor. ${ }^{[5 a]}$ Yersin presented brightly luminescent and $\mathrm{O}_{2}$-sensitive $\mathrm{Pt}^{\prime \prime}$ cyclometalated with carborane. ${ }^{[5 e]}$ Photosensitized luminescence of lanthanide complexes is also attractive for PSPs. ${ }^{[6]}$ Amao presented the first PSP using luminescent Eu $u^{\text {III }}$ complex based on the $\mathrm{O}_{2}$-quenching in the $\mathrm{T}_{1}$ state of the photosensitized ligand. [6a] We have reported oxygen-sensitive near-infrared luminophores composed of porous $\mathrm{Yb}^{\text {III }}$ coordination porous networks for PSPs. ${ }^{[6 b]}$ The $\mathrm{O}_{2}$-sensitive metal complex is expected to be useful for PSP applications in aerodynamics.

Recently, time-resolved analysis using the emission decay profile of phosphorescent materials has been a focus for fluid dynamics, aeronautical and environmental engineering ${ }^{[7]}$ The emission lifetime-based PSP systems have shown significant potential for unsteady flow diagnostics of aircrafts, high-speed vehicles and turbomachinery. ${ }^{[8]}$ Environment-visualized analysis using time-resolved phosphorescence $(>\mu \mathrm{s})$ can also remove some short-lifetime luminescent noises on the luminescent surfaces such as organic light-emitting devices (OLEDs) and biomedical applications. ${ }^{[9]}$ However, general transition-metal complexes lead to a significant decrease of their phosphorescence lifetimes in the presence of small amounts of $\mathrm{O}_{2}$, which results in the formation of non-emission compounds in air. According to the luminescent lanthanide complexes, their $4 f$ $4 f$ emission lifetimes are independent of $\mathrm{O}_{2}$-quenching in the $\mathrm{T}_{1}$ state of the photosensitizer. ${ }^{[6 \mathrm{~b}]}$ Novel time-resolved analysis of phosphorescence under a highly concentration of $\mathrm{O}_{2}$ environments is thus required.

We herein select an Eu $u^{\text {III }}$ coordination compound attached with triphenylene units for the back energy transfer (BEnT) process. The BEnT process with activation energy of the luminescence could be a key point for construction of a novel time-resolved $\mathrm{O}_{2}-$ analysis.[10] In our previous study, an Eu'll complex with triphenylene phosphine-oxide, $\left[\mathrm{Eu}(\mathrm{hfa})_{3}(\mathrm{dppto})_{2}\right]$ (hfa: hexafluoroacetyl-acetonate, dppto: 2-diphenylphosphoryltriphenylene), had a long $T_{1}$ lifetime on a low-lying energy level $\left(20,490 \mathrm{~cm}^{-1}\right)^{[11]}$ The low-lying energy level of the photosensitization ligand is much close to that of the emitting level of the Eu $u^{\text {III }}$ ion $\left({ }^{5} \mathrm{D}_{0}: 17,200 \mathrm{~cm}^{-1}\right)$, which promotes BEnT with activation energy from the excited Eu'll complex. Multi-dentate coordination sites should also be attached in the photosensitization triphenylene unit for the formation of a thermostable structure. ${ }^{[6 b]}$ The multidentate coordination sites are expected to construct the thermo-stable tight packing networks and an $\mathrm{O}_{2}$-active porous structure in the Eu' ${ }^{\text {III }}$ coordination polymer.

On the basis of the two criteria for an advanced pressuresensitive luminophore, a novel Eu III coordination polymer with

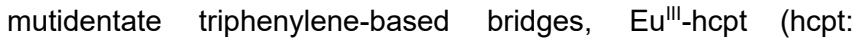
2,3,6,7,10,11-hexakis(4-carboxyphenyl)triphenylene) with the photosensitized energy transfer (EnT), back energy transfer 
(BEnT) and $\mathrm{O}_{2}$-quenching $\left(\mathrm{O}_{2}-\mathrm{Q}\right)$ processes is here designed (Figs. 1a and b). The hcpt possesses benzene-spacers for the construction of a porous structure of a three-dimension networks. ${ }^{[12]}$ The Eulll coordination polymer with hcpt networks successfully exhibited $\mathrm{O}_{2}$-dependent emission lifetimes linked with the BEnT process (decrease of non-radiative rate constant, $k_{n r}$ ). The photophysical parameters of previously reported Eu $\mathrm{u}^{\prime \prime \prime}$ complexes, with or without triphenylene units, $\left[\mathrm{Eu}(\mathrm{hfa})_{3}(\mathrm{dppto})_{2}\right]$ or $\left[\mathrm{Eu}(\mathrm{hfa})_{3}(\mathrm{tppo})_{2}\right]$ (tppo: triphenylphosphine oxide), were also estimated as standard references (Figs. 1c and d). In this study, an advanced pressure-sensitive luminophore for aerodynamics applications is demonstrated.

a) UV

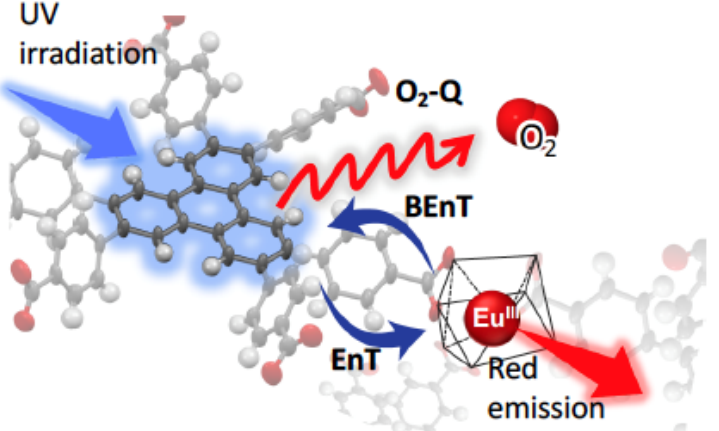

b)

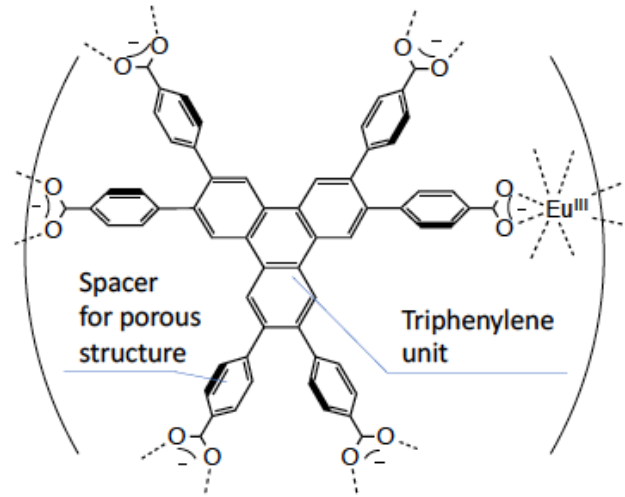

c)

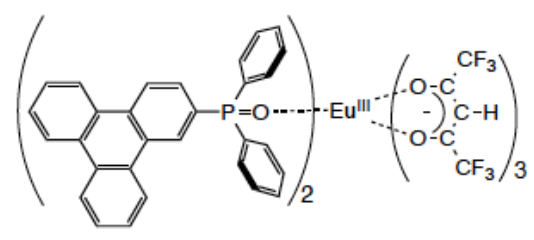

d)

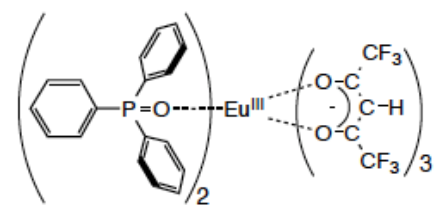

Figure 1. a) Schematic representation of Eu'll coordination polymers with photosensitized energy transfer (EnT), back energy transfer (BEnT), and $\mathrm{O}_{2}$ quenching process $\left(\mathrm{O}_{2}-\mathrm{Q}\right)$. Figure Images based on the $\mathrm{X}$-ray single crystal data (CCDC1424089. Chemical structures of b) Eu'll-hcpt c) [Eu(hfa $\left.)_{3}(\text { dppto })_{2}\right]$, and d) $\left[\mathrm{Eu}(\mathrm{hfa})_{3}(\mathrm{tppo})_{2}\right]$
The precursor hexakis(4-methoxycarbonylphenyl)triphenylene (hmcpt) was prepared by the boron-coupling reaction of 2,3,6,7,10,11-hexabromo-triphenylene with 4-methoxycarbonylphenyl) boronic acid, a $\mathrm{Pd}$ catalyst, and $\mathrm{Na}_{2} \mathrm{CO}_{3}$ in toluene/dioxane for $46 \mathrm{hrs}$, according to our previous reported paper. ${ }^{[12]}$ The hexadentate triphenylene unit hcpt-H (hexakis(4carbonylphenyl)triphenylene) was synthesized by the hydrolysis reaction of hmcpt with $\mathrm{KOH}$ in THF/water (CCDC1424089). ${ }^{[2]}$ The Eu'll coordination polymer Eu'll-hcpt was obtained by the reaction of $\mathrm{Eu}\left(\mathrm{NO}_{3}\right)_{3} \cdot 6 \mathrm{H}_{2} \mathrm{O}$ with hcpt-H and triethylamine in $\mathrm{NN}-$ dimethylacetoamide (DMA) at $130^{\circ} \mathrm{C}$ for $72 \mathrm{hrs}$.

The crystalline structure of Eu'll-hcpt was characterized using IR and XRD measurements (XRD data: Fig. 2a, IR data: Supporting Information Fig. S1). The IR stretching signal of carbonyl groups in Eu'II-hcpt $\left(1536 \mathrm{~cm}^{-1}\right)$ was smaller than that in the hcpt-H ligand $\left(1693 \mathrm{~cm}^{-1}\right)$. The signal shift is due to the formation of carboxylate groups with Eu III ions. The XRD pattern of Eu(hcpt) $]_{m}$ (Figs. 2a-ii) is highly different from that of hcpt-H (Figs. 2a-i). The average crystal grain size from the XRD signal at $8.7^{\circ}$ was calculated to be $23 \mathrm{~nm}$. The XRD patterns of the prepared Eu'I'-hcpt (Fig. 2a-ii) agrees well with that of Eu'l'-hcpt after heat treatment at $200^{\circ} \mathrm{C}$ and $300^{\circ} \mathrm{C}$ (Figs.2a-iii and iv). The decomposition temperature using thermal gravimetric analysis was estimated to be $420^{\circ} \mathrm{C}$ (see Supporting Information Fig. S2).
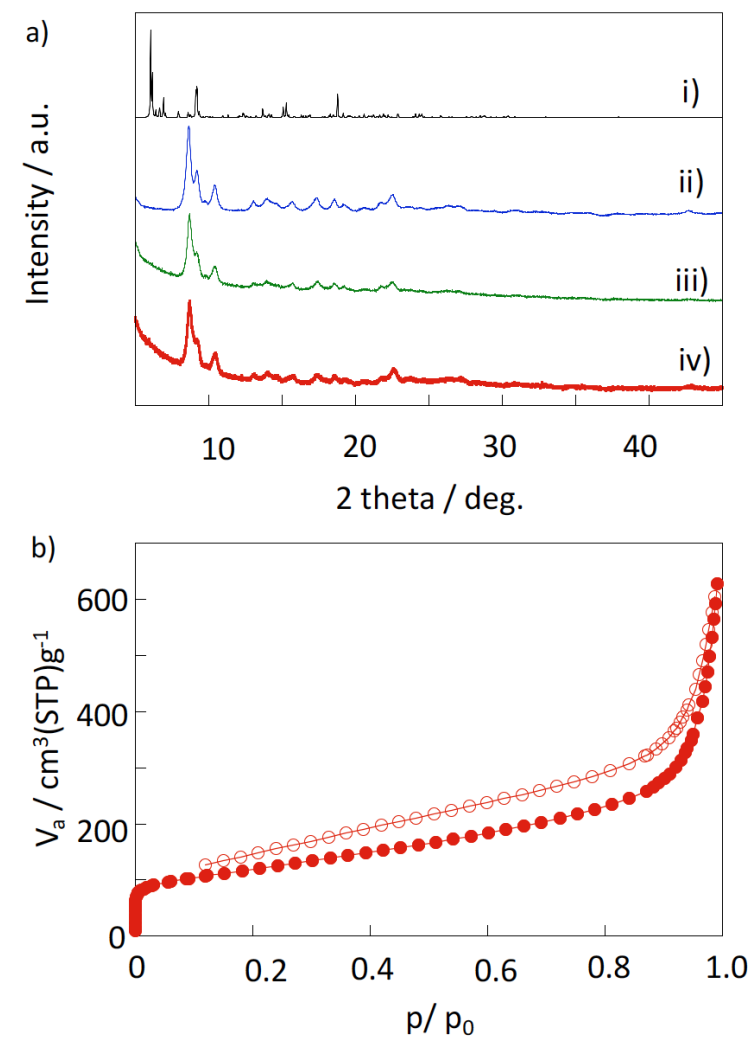

Figure 2. a) XRD profiles of i) hcpt from cif file (CCDC1424089), ii) prepared

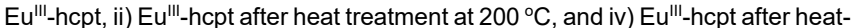
treatment at $300^{\circ} \mathrm{C}$. b) Nitrogen adsorption isotherm of Eu'l'-hcpt measured after heat-treatment at $200^{\circ} \mathrm{C}$ for $6 \mathrm{hrs}$. 
The decomposition temperature of Eu'II-hcpt is much higher than those of previous Eulll coordination polymers with bidentate and tridentate phosphine oxides $\left(<360^{\circ} \mathrm{C}\right)$. The hexadentate carboxylic acids play an important role in the formation of tightpacked three-dimensional networks in Eu'II-hcpt, producing hyper thermo-stable ability.

The gas adsorption capacity of Eu'll-hcpt was estimated by measuring its nitrogen adsorption isotherm measurements, as shown in Fig. $2 \mathrm{~b}$. The difference between the adsorption and desorption rate constants for this material produced hysteresis in the isotherm. The effective increase in the plot at $p / p_{0} \approx 0$ was attributed to the adsorption of gas in the nanopores. Using the ideal gas law, the amount nitrogen adsorption amount at a pressure $(P)$ of $101.25 \mathrm{kPa}$ and a temperature $(T)$ of $273.15 \mathrm{~K}$ (that is, STP conditions) was calculated using the ideal gas constant (R) of $8.31 \mathrm{~J} \mathrm{~K}^{-1} \mathrm{~mol}^{-1}$ and a volume $(\mathrm{V})$ of $64.69 \mathrm{~cm}^{3}$ $\left(\mathrm{p} / \mathrm{p}_{0}=1.0\right)$. The amount of nitrogen adsorption was evaluated to be $8.4 \times 10^{-2} \mathrm{~cm}^{3} \mathrm{~g}^{-1}$, which is similar to that of previously reported porous $\mathrm{Yb}^{\text {III }}$ coordination polymer with octadentate pyrene units $\left(8.0 \times 10^{-2} \mathrm{~cm}^{3} \mathrm{~g}^{-1}\right)$. ${ }^{[6]}$ The specific surface area of Eull'-hcpt was estimated using Brunauer-Emmet-Teller (BET) calculation as 407 $\mathrm{m}^{2} \mathrm{~g}^{-1}$ (Fig. S3). Overall, these results indicate that Eu'll-hcpt has a three-dimensional network structure and nano-sized porous cavities suitable for $\mathrm{O}_{2}$ adsorption.

The Eu'll-hcpt exhibited strong luminescence under UV irradiation in the solid state $\left(\lambda_{e x}=340 \mathrm{~nm}\right.$, Fig. 3 red line and inset picture). The emission bands observed at $578,590,614,652$, and $700 \mathrm{~nm}$ were assigned to be $4 f-4 f$ transitions of ${ }^{5} \mathrm{D}_{0} \rightarrow{ }^{7} \mathrm{~F}_{J}(J=0,1$, 2,3 , and 4) in Eu'lli ions. The broad excitation bands at around 380 $\mathrm{nm}$ were contributed to the $\pi-\pi^{*}$ transition of the hcpt ligand, indicating effective photosensitized energy transfer from hcpt to Eu"l ions in the Eu'll coordination polymer. The sharp excitation bands at 393,463 and $534 \mathrm{~nm}$ were assigned to the ${ }^{7} \mathrm{~F}_{0} \rightarrow{ }^{5} \mathrm{~L}_{6},{ }^{7} \mathrm{~F}_{0}$ $\rightarrow{ }^{5} D_{2}$ and ${ }^{7} F_{0} \rightarrow{ }^{5} D_{1}$ transitions of Eulll ions, respectively.

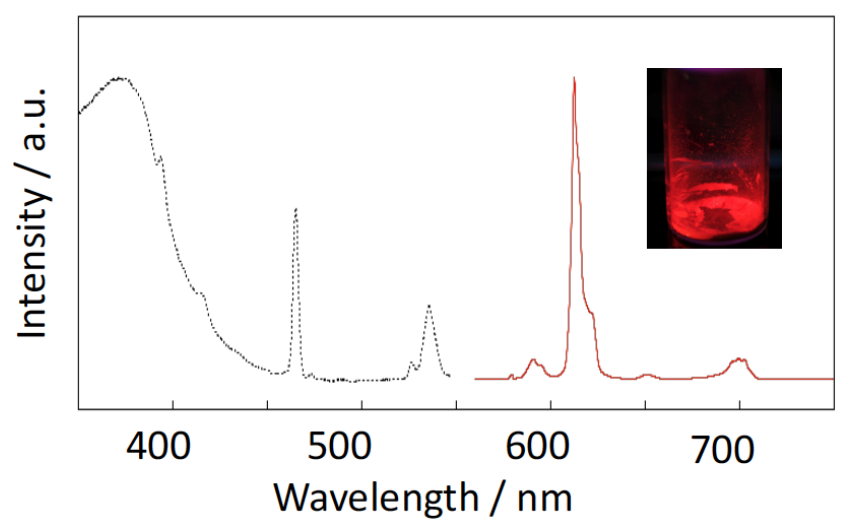

Figure 3. Excitation (black dot line) and emission spectra (red line) of Eu'll-hcpt in solid state under air at $300 \mathrm{~K}$. Excitation wavelength for emission spectrum: $340 \mathrm{~nm}$. Emission wavelength for excitation spectrum: $610 \mathrm{~nm}$. Inset figure is image of red luminescence of Eu'll'-hcpt under air.
The luminescence intensity of Eu'll-hcpt under vacuum is much larger than that in air. Emission lifetime measurements were conducted under vacuum, argon ( $\mathrm{Ar})$, air, $\mathrm{Ar}-\mathrm{O}_{2}(1: 1 \mathrm{v} / \mathrm{v})$, and $\mathrm{O}_{2}$ environments to quantitatively estimate the $\mathrm{O}_{2}$-quenching efficiency. Their emission lifetimes were gradually decreased with an increase in $\mathrm{O}_{2}$ concentration (Figs. $4 \mathrm{a}$ and b, relative emission spectra: supporting information Fig. S8). The emission lifetime of Eu'll-hcpt under vacuum ( $\left.\tau_{\mathrm{Eu}} 0.86 \mathrm{~ms}\right)$ was twice as large as that under $\mathrm{O}_{2}\left(\tau_{E u}=0.48 \mathrm{~ms}, 1\right.$ atom:101.3 kPa). The Stern-Volmer plot was dependent on the $\mathrm{O}_{2}$ concentration and showed a pseudo-linear correlation $\left(\tau_{0 \%} / \tau_{100 \%}=1.81\right.$, see Supporting Information Fig. S4). The gradual lifetime-response in high $\mathrm{O}_{2}$ concentration for aerodynamics was successfully observed.

The photophysical parameters and time-decay profiles of Eu'IIhcpt are summarized in Table 1. The gradual decrease of the emission lifetime of Eu'l'-hcpt is caused by the increase of the nonradiative rate constant $k_{\mathrm{nr}}$. On the other hand, radiative rate constant $k_{\mathrm{r}}$ of Eu'll-hcpt under vacuum is similar to those under $\mathrm{Ar}$, air, $\operatorname{Ar}-\mathrm{O}_{2}(1: 1)$, and $\mathrm{O}_{2}$ conditions. In the tight-packed Eu'II-hcpt system, the coordination geometry related to $k_{r}$ could be independent of the surrounding environment. The emission quantum yield $\Phi_{4 f-4 f}$ of Eu'll-hcpt under vacuum was calculated to be $70 \%$, which is similar to those of bright luminescent $\left[\mathrm{Eu}(\mathrm{hfa})_{3}(\mathrm{dppto})_{2}\right]$ and $\left[\mathrm{Eu}(\mathrm{hfa})_{3}(\mathrm{tppo})_{2}\right]^{\left[{ }^{[11]}\right.}$ The large $\Phi_{4 f-4 f}$ of Eu'l'hcpt is also due to the small non-radiative rate constant for the tight-packing structure in this solid. The emission lifetimes of $\left[\mathrm{Eu}(\mathrm{hfa})_{3}(\mathrm{dppto})_{2}\right]$ and $\left[\mathrm{Eu}(\mathrm{hfa})_{3}(\mathrm{tppo})_{2}\right]$ under vacuum are similar with those measured in air. These results indicate that the nanoporous structure of Eull'-hcpt plays a role in the $\mathrm{O}_{2}$-sensing properties.

The gradual decrease of the emission lifetime in $\mathrm{O}_{2}$ could be directly linked to the BEnT in Eu'll-hcpt. The ligandphosphorescence of the $\mathrm{Gd}^{\text {III }}$ coordination polymer with hcpt ligands was therefore measured to analyze the BEnT process.
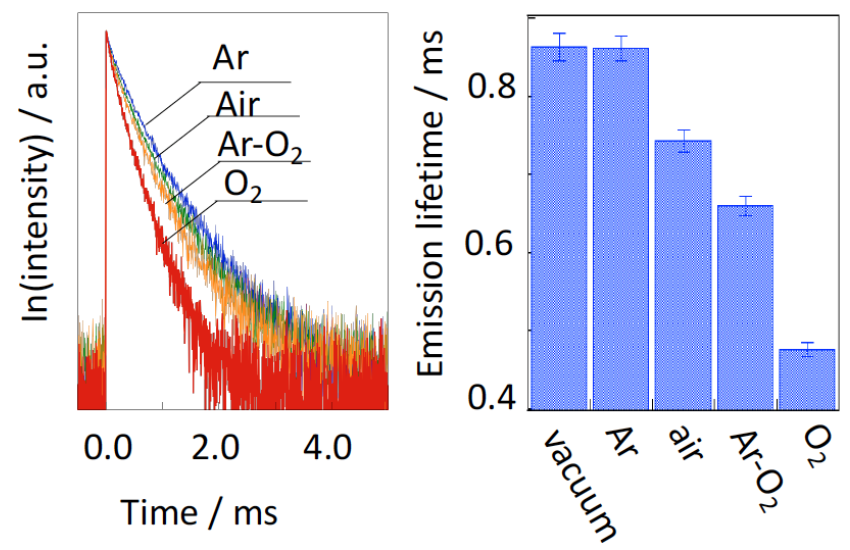

Figure 4. a) Emission decay profiles and b) histograms of the emission lifetime of Eu'll-hcpt in solid state under vacuum, $\mathrm{Ar}$, air, $\mathrm{Ar}-\mathrm{O}_{2}(1: 1)$ and $\mathrm{O}_{2}$ atmosphere. Excitation source: Nd: YAG (wavelength: $355 \mathrm{~nm}(3 \omega)$, plus width $10 \mathrm{~ns})$. 


\begin{tabular}{|c|c|c|c|c|c|}
\hline sample & condition & $\begin{array}{l}\tau_{E E^{[a]}}^{[a]} \\
/ \mathrm{ms}\end{array}$ & $\begin{array}{c}\Phi_{4 f-4 f^{[\mathrm{b}]}} \\
/ \%\end{array}$ & $\begin{array}{l}k_{r}{ }^{[b]} \\
/ \mathrm{s}^{-1}\end{array}$ & $\begin{array}{l}k_{n n}[\mathrm{~b}] \\
/ \mathrm{s}^{-1}\end{array}$ \\
\hline \multirow[t]{5}{*}{ Eu'll-hcpt } & vacuum & $0.86 \pm 0.02$ & 70 & $8.1 \times 10^{2}$ & $3.5 \times 10^{2}$ \\
\hline & $\mathrm{Ar}$ & $0.86 \pm 0.01$ & 69 & $8.0 \times 10^{2}$ & $3.6 \times 10^{2}$ \\
\hline & air & $0.74 \pm 0.01$ & 56 & $7.7 \times 10^{2}$ & $6.0 \times 10^{2}$ \\
\hline & $\mathrm{Ar}-\mathrm{O}_{2}$ & $0.66 \pm 0.01$ & 52 & $7.9 \times 10^{2}$ & $7.3 \times 10^{2}$ \\
\hline & $\mathrm{O}_{2}$ & $0.48 \pm 0.01$ & 37 & $7.8 \times 10^{2}$ & $1.3 \times 10^{3}$ \\
\hline \multirow[t]{2}{*}{$\begin{array}{l}{\left[\mathrm{Eu}(\mathrm{hfa})_{3}\right.} \\
\left.\text { (dppto })_{2}\right]\end{array}$} & vacuum & $0.71 \pm 0.06$ & 73 & $1.0 \times 10^{3}$ & $3.8 \times 10^{2}$ \\
\hline & air & $0.69 \pm 0.06$ & 71 & $1.0 \times 10^{3}$ & $4.2 \times 10^{2}$ \\
\hline \multirow[t]{2}{*}{$\begin{array}{l}{\left[\mathrm{Eu}(\mathrm{hfa})_{3}\right.} \\
\left.(\mathrm{tppo})_{2}\right]\end{array}$} & vacuum & $0.71 \pm 0.05$ & 76 & $1.1 \times 10^{3}$ & $3.3 \times 10^{2}$ \\
\hline & air & $0.70 \pm 0.05$ & 76 & $1.1 \times 10^{3}$ & $3.4 \times 10^{2}$ \\
\hline
\end{tabular}

[a] The excitation wavelength: $355 \mathrm{~nm}$ ( $3 \omega$ for $\mathrm{Nd}$ : YAG). The $T_{\text {Eu }}$ were determined by fitting analyses of emission decay profiles. [b] Calculated using equations $1-5$ in supporting information.

The $\mathrm{Gd}^{\text {IIII }}$ complex without the $4 \mathrm{f}-4 \mathrm{f}$ transition in the visible region is well-known as a typical compound for estimation of the $T_{1}$ level of the organic ligand. The $T_{1}$ level of $\mathrm{Gd}^{\prime \prime \prime}$-hcpt estimated using phosphorescence spectra with vibrational Gaussian fitting curves was calculated to be $19,000 \mathrm{~cm}^{-1}$ (Fig. 5). The $T_{1}$ level is much close to that of the ${ }^{5} D_{0}$ emitting level of the Eu $u^{\prime \prime \prime}$ ion $\left(17,200 \mathrm{~cm}^{-1}\right)$. The energy gap between excited $T_{1}$ and ${ }^{5} D_{0}$ levels is smaller than the acceptable gap for BEnT in reported previously $(<1,850 \mathrm{~cm}$ 1). ${ }^{[13]}$ The phosphorescence time decay profile of $\mathrm{Gd}^{\text {III-hcpt }}$ showed two emissive components with long decay profiles (120 ms and 2000 ms: see Supporting Information Fig. S5). The long decay phosphorescence promotes an effective quenching process for $\mathrm{O}_{2}$-sensing properties $\left(\mathrm{O}_{2}-\mathrm{Q}\right.$ process). The activation energy from the EullI ion to $T_{1}$ state was also calculated by Arrhenius analysis (Supporting Information Fig. S6). The calculated activation energy $E_{\mathrm{a}}$ at around room temperature was $3.6 \times 10^{3} \mathrm{~cm}^{-1}$. This calculated parameter is similar to that of thermo-sensitive $\left[\mathrm{Tb}(\mathrm{hfa})_{3}(\mathrm{tppo})_{2}\right]$ at room temperature $\left(E_{\mathrm{a}}=\right.$ $\left.3.0 \times 10^{3} \mathrm{~cm}^{-1}\right) \cdot{ }^{[10]}$ The Arrhenius analysis indicates that the BEnT from Eu $u^{\prime \prime \prime}$ to the $T_{1}$ state should be activated at room temperature. We consider that the gradual decrease of emission lifetime is caused by the BEnT process in Eu'll-hcpt.

Figure $5 b$ shows a schematic diagram with a summary of the EnT, BEnT and $\mathrm{O}_{2}-\mathrm{Q}$ processes. The Arrhenius plot of the emission quantum yields of Eu'll-hcpt in solid under vacuum is shown in Figure S6. The emission spectral shape of lanthanide complex is generally affected by coordination geometry. The crystal phase transition of Eu $u^{\prime \prime \prime}$-hcpt under $400{ }^{\circ} \mathrm{C}$ was not observed. The spectral shape of lanthanide complex was not also depended on the temperature. From these results, the temperature-depended luminescence intensity is related to the non-radiative rate constant, the emission quantum yield and the emission lifetime. On the other hand, the oxygen-depended emission lifetime of Eu'll complex with triphenylene phosphineoxide (dppto) was not observed, although triplet level of dppto $\left(20490 \mathrm{~cm}^{-1}\right)$ is much similar to that of hcpt ligands $\left(19000 \mathrm{~cm}^{-1}\right)$ The reference compounds $\left[\mathrm{Eu}(\mathrm{hfa})_{3}(\mathrm{dppto})_{2}\right]$ in $\mathrm{CH}_{2} \mathrm{Cl}_{2}(1 \mathrm{mM})$ showed small oxygen-quenching of the emission (see supporting information Fig. S9). The activation energy for back energy transfer of $\left[\mathrm{Eu}(\mathrm{hfa})_{3}(\mathrm{dppto})_{2}\right]$ in $\mathrm{CH}_{2} \mathrm{Cl}_{2}$ should be larger than that of Eu-hcpt in solid. The specific LC band of Eu'll-hcpt was not observed. From these photophysical aspects, effective oxygen process of Eu-hcpt might be produced by not only small $E_{a}$, but also the porous structure.

In conclusion, we successfully synthesized a luminescent Eu'II coordination polymer with hexadentate triphenylene porous units. The Eu-hcpt is a metal-coordination polymer, which is prepared by the chemical equilibrium in methanol solution. The synthetic process and three-dimensional coordination bonds in Eu-hcpt provide insoluble and tight-packing white powder, which is much different from normal organic polymer linked with covalent bonds. The crystalline and porous structural data of Eu-hcpt were shown in Fig. 2 (a: XRD, b: Nitrogen adsorption isotherm data) and Fig. S3 (BET data). The $\mathrm{O}_{2}$-pressure sensing properties of Eu'll-hcpt were also examined at highly temperature $\left(127{ }^{\circ} \mathrm{C}\right.$, See Supporting Information Fig. S7). The thermo-stable structure of Eu'll-hcpt (decomposition temperature $\approx 420{ }^{\circ} \mathrm{C}$ ) promotes effective $\mathrm{O}_{2}$-pressure sensing at high temperature, although previous PSPs (pressure sensitive paints) perform under $70^{\circ} \mathrm{C}$. The $\mathrm{O}_{2}$-pressure sensing ability of Eu'll-hcpt is based on the a novel photophysical mechanism with the BEnT process.

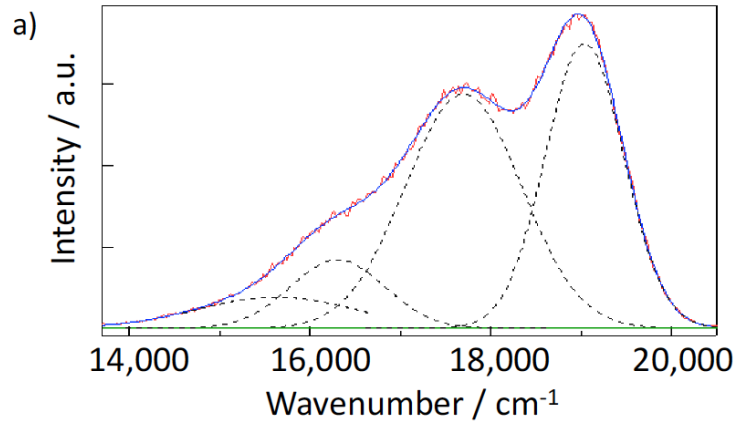

b)

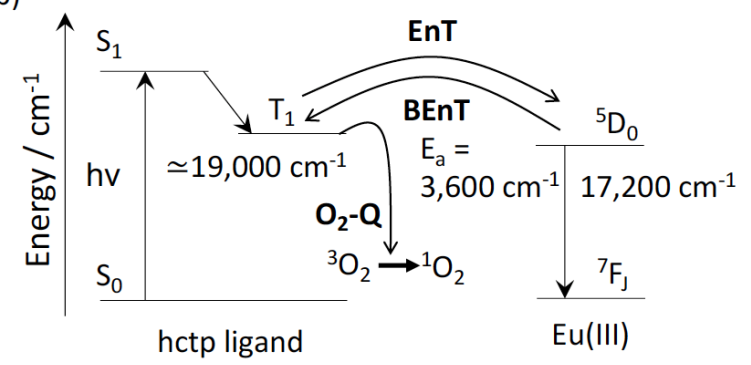

Figure 5. a) Phosphorescence spectra with vibrational Gaussian fitting curves of Gd"I-hcpt in solid state at $77 \mathrm{~K}$. Red line: phosphorescence spectrum, Blue and black dot lines: Gaussian fitting functions. Green line: difference between phosphorescence spectrum and Gaussian functions. b) The schematic diagram of the back energy transfer (BEnT) from ${ }^{5} \mathrm{D}_{0}$ emitting level of Eull' to $T_{1}$ state of hcpt ligand in Eu'II-hcpt. 
The advanced PSPs in the high temperature region are expected to be useful for not only for the body design of future aircraft and spaceship, but also for research on submarine volcanoes under the sea. ${ }^{[1 \mathrm{c}]}$ This molecular design of an $\mathrm{O}_{2-}$ pressure sensor for aerodynamics analysis in this study is significantly different from the low level of $\mathrm{O}_{2}$-detection using previously reported metal complexes in liquid media. We have also reported color-changeable Temperature Sensitive paint (TSP) using Eu ${ }^{\prime \prime \prime} / \mathrm{Tb}^{\prime \prime \prime}$ coordination polymers known as chameleon luminophores. ${ }^{[14,15]}$ The combination of PSPs and TSPs would also be useful for architecture designs and bio-medical applications. ${ }^{[16,17]}$ Advanced PSPs are thus expected to open-up a frontier field between photophysical science, molecular chemistry and fluid engineering.

\section{Acknowledgements}

We appreciate Prof. Hajime Ito and Dr. Tomohiro Seki., Faculty of Engineering, Hokkaido University for temperature-depended XRD measurements. This work was supported by JSPS KAKENHI Grant Number JP18H04497. This work was also partially supported by two Grants-in-A.id for Scientific Research $(18 \mathrm{H} 02041$ and $18 \mathrm{H} 04496)$

Keywords: coordination polymer • energy transfer • europium • luminescence $\cdot$ oxygen sensor

[1] a) B. G. McLachlan, J. H. Bell, Exp. Therm. Fluid Sci. 1995, 10, 470485; b) X. Wang, O. O. Wolfbeis, Chem. Soc. Rev. 2014, 43, 3666-3761. C) M. Schäferling, Angew. Chem, Int. Ed. 2012, 51, 3532.

[2] M. Eberhart, S. Löhle, A. Steinbeck, T. Binder, S. Fasoulas, Atmos. Meas. Tech. 2015, 8, 3701-3714.

[3] M. Anyoji, D. Numata, H. Nagai, K. Asai, J. Vis. 2015, 18, 297-309.

[4] E. Mei, S. Vinogradov, R. M. Hochstrasser, J. Am.Chem.Soc. 2003, 125, 13198-13204

[5] a) K. S. Choung, K. Marroquin, T. S. Teets, Chem. Sci. 2019, 10, 5124-
5132; b) P. Irmler, R. F. Winter, Dalton. Trans. 2016, 45, 10420-10434 c) S. M. Borisov, A. Alemayehu, A. Ghosh, J. Mater.Chem. C 2016, 4 5822-5828. d) Y. Chen, C. Lin, G. Kee, M. Ho, CrystEngComm. 2015 2129-2140. e) A. M. Prokhorov, T. Hofbeck, R. Czerwieniec, A. F. Suleymanova, D. N. Kozhevnikov, H. Yersin, J. Am. Chem.Soc. 2014, 136, 9637-9642. f) C. Lin, C. Chen, S. K. Kundu, J. Yang, Inorg. Chem. 2014, 53, 737-745

[6] a) Y. Amao, I. Okura, T. Miyashita, Bull. Chem. Soc. Jpn. 2000, 73, 2663 2668; b) Y. Hasegawa, T. Matsui, Y. Kitagawa, T. Nakanishi, T. Seki, H. Ito, Y. Nakasaka, T. Masuda, K. Fushimi, Chem. Eur. J. 2019, 25, 1230812315.

[7] A. Weiss, R. Geisler, T. Schwermer, D. Yorita, U. Henne, C. Klein, M. Raffel, Exp. Fluids 2017, 58, 120.

[8] D. Peng, L. Jiao, Y. Yu, Y. Liu, T. Oshio, T. Kawakubo, A. Yakushiji, exp. Fluids 2017, 58, 127.

[9] a) L. M. Hirvonen, Z. Petrášek, A. Beeby, K. Suhling, New. J. Phys. 2015, 17, 023032.; b) L. Pohl, Z. Kohári, A. Poppe, Microelectronics Reliability 2018, 85, 198-206; c) M. M. Azain, M. R. Mansor, G. Omar, S. H. S. M. Fadzullah, S. R. Esa, L. M. Lim, D. Sivakumar, M. N. A. Nordin, Synthetic Mat. 2019, 247, 191-201.

[10] M. Yamamoto, Y. Kitagawa, T. Nakanishi, K/ Fushimi, Y. Hasegawa Chem. Eur. J. 2018, 24, 17719-17726.

[11] Y. Kitagawa, F. Suzue, T. Nakanishi, K. Fushimi, Y. Hasegawa, Dalton Trans. 2018, 47, 7327-7332.

[12] I. Hisaki, N. Ikenaka, N. Tohnai, M. Miyata, Chem. Commun. 2016, 52, 300-302.

[13] M. Latva, H. Takalo, V.-M. Mukkala, C. Matachescu, J. C. RodriguezUbis, J. Kankare, J. Lumin. 1997, 75, 149-169.

[14] K. Miyata, Y. Konno, T. Nakanishi, A. Kobayashi, M. Kato, K. Fushimi, Y. Hasegawa, Angew. Chem. Int. Ed. 2013, 52, 6413-6416.

[15] M. Hatanaka, Y. Hirai, Y. Kitagawa, T. Nakanishi, Y. Hasegawa, K. Morokuma, Chem. Sci. 2017, 8, 423-429.

[16] N. Bai, L. Wang, Q. Wang, Y. Wang, P. Lu, G. Li, Y. Zhang, j. Yang, K. Zie, X. Zhao, C. F. Guo, Nature Comm. 2020, 11, 209.

[17] D. Hara, H. Komatsu, A. Son, S. Nishimoto, K. Tanabe, Bioconjugate Chem. 2015, 26, 645-649. 


\section{Entry for the Table of Contents}

Layout 1:

\section{COMMUNICATION}

Oxygen-sensitive Eu $\quad$ Eu coordination polymers with strong luminescence properties under air was successfully fabricated by introducing hexadentate tight and porous three-dimensional networks. The $\mathrm{O}_{2}$-sensing property is based on the back energy transfer with activation energy. The $\mathrm{O}_{2}$-sensitive coordination polymers have potential application in aerodynamic experiments.

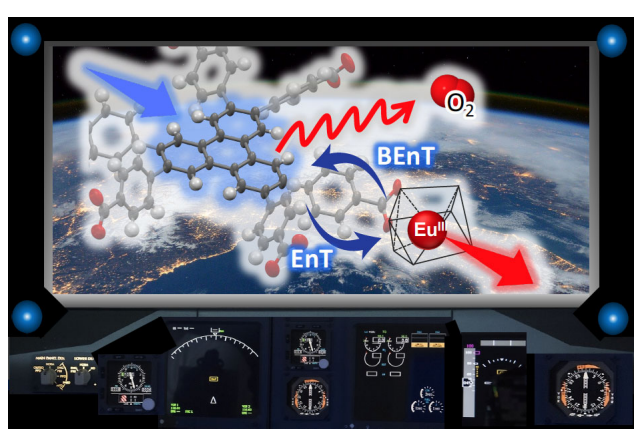

Yasuchika Hasegawa, * Takuya Sawanobori, Yuichi Kitagawa, Koji Fushimi, Yuta Nakasaka, Takao Masuda, Ichiro Hisaki

Page No. - Page No.

An Europium(III) Luminophore with Pressure-sensing Units: Effective Back Energy Transfer in Coordination Polymers with Hexadentate Porous StableNetworks 